\title{
Translocation Breakpoint
}

National Cancer Institute

\section{Source}

National Cancer Institute. Translocation Breakpoint. NCI Thesaurus. Code C13660.

The point on a chromosome where the normal DNA sequence is juxtaposed with DNA

from another chromosome. Usually expressed as a band number designation (eg. t(9;22)

(q34;q11) Philadelphia Chromosome) 